九州大学学術情報リポジトリ

Kyushu University Institutional Repository

\title{
Supervised image classification based on AdaBoost with Contextual Weak Classifiers
}

Nishii, Ryuei

Kyushu University, Graduate School of Mathematics

Eguchi, Shinto

Institute of Statistical Mathematics

http://hdl. handle. net/2324/3350

出版情報：MHF Preprint Series. MHF2004-21，2004-07-07. 九州大学大学院数理学研究院 バージョン：

権利関係 : 


\title{
MHF Preprint Series
}

Kyushu University

21st Century COE Program

Development of Dynamic Mathematics with

High Functionality

\section{Supervised image classification based on AdaBoost with contextual weak classifiers}

\author{
R. Nishii \& S. Eguchi
}

MHF 2004-21

( Received July 7, 2004 )

Faculty of Mathematics

Kyushu University

Fukuoka, JAPAN 


\title{
Supervised Image Classification based on AdaBoost with Contextual Weak Classifiers
}

\author{
Ryuei NISHII \\ Kyushu University \\ Graduate School of Mathematics, Hakozaki, Higashiku, Fukuoka 812-8581, Japan \\ Email: nishii@math.kyushu-u.ac.jp \\ Shinto EGUCHI \\ Institute of Statistical Mathematics \\ Minami-azabu, Minatoku, Tokyo 106-8569, Japan \\ Email: eguchi@ism.ac.jp
}

\begin{abstract}
AdaBoost, one of machine learning techniques, is employed for supervised classification of land-cover categories of geostatistical data. We introduce contextual classifiers based on neighboring pixels. First, posterior probabilities are calculated at all pixels. Then, averages of the posteriors in various neighborhoods are calculated, and the averages are used as contextual classifiers. Weights for the classifiers can be determined by minimizing the empirical risk with multiclass. Finally, a linear combination of classifier is obtained. The proposed method is applied to artificial multispectral images and shows an excellent performance similar to the MRF-based classifier with much less computation time.
\end{abstract}

\section{Introduction}

AdaBoost proposed by [0] is one of machine learning techniques and has been progressed for pattern recognition rapidly and widely. AdaBoost combines weak classifiers into a weighted voting machine, and it shows high performance in various fields, see [2-4].

In this paper, we introduce AdaBoost for contextual image classification for geostatistical data. First, we prepare a posterior probability given feature vector at each pixel. The posteriors can be defined by machine learning techniques as well as statistical methods. Then, averages of the posteriors are calculated in various types of neighborhoods, and we positively use them as classifiers. The weights for the classifiers are obtained by minimizing the empirical risk with multiclass. Finally, a linearly combined classifier gives a contextual voting machine.

The proposed method is shown to be a very fast algorithm. It needs only $1 / 100$ of time required by MRF-based classifiers implemented by [?]. The performance of our method keeps equivalent to that of MRF-based classifiers. Also, the proposed method is very flexible since the posteriors can be derived from various techniques: support vector machines (SVM), AdaBoost, and artificial neural networks (ANN), see e.g. [6-8].

In Section II, Real AdaBoost is explained. The determination of the coefficient of 
classifiers are discussed in a multiclass case. Section III defines contextual classifiers. Neighborhoods of a pixel and the averages of the posteriors in the neighborhoods are defined, which will be viewed as classifiers. The proposed method is applied to a numerical example in Section IV. It shows an excellent performance similar to MRF-based classifiers. Section V concludes the paper.

\section{Real AdaBoost with multiclass}

Let $\mathcal{D}=\{1, \ldots, n\}$ be a training area consisting of $n$ pixels, and each pixel belongs to one of $g$ possible categories $C_{1}, \ldots, C_{g}$. Suppose that an $m$-dimensional feature vector $\boldsymbol{x}_{i} \in \mathbb{R}^{m}$ is observed at each pixel $i$. A label of the category covering the pixel $i$ is denoted by $y_{i} \in\{1, \ldots, g\}$. Let $f(\boldsymbol{x}, y)$ be a discriminant function. We allocate a feature vector $\boldsymbol{x} \in \mathbb{R}^{m}$ into a category label:

$$
y_{f}(\boldsymbol{x})=\underset{y \in\{1, \ldots, g\}}{\arg \max } f(\boldsymbol{x}, y) .
$$

In the ordinary setting of AdaBoost, the function $f$ is restricted to take only zero or one as follows.

$$
f(\boldsymbol{x}, y)= \begin{cases}1 & \text { if } y=y_{f}(\boldsymbol{x}) \\ 0 & \text { otherwise }\end{cases}
$$

Typical weak classifiers are decision stumps given by the functions $\delta \operatorname{sign}\left(x^{j}-t\right)$ in binary class with label set $\{-1,1\}$, where $\delta= \pm 1, t \in \mathbb{R}, x^{j}$ is the $j$-th variate of the feature vector $\boldsymbol{x}$ and $\operatorname{sign}(z)$ is the sign of the $\operatorname{argument} z$.

We also consider the case such that the discriminant function $f$ takes real values. For example, a posterior probability:

$$
p(y \mid \boldsymbol{x})=p(\boldsymbol{x} \mid y) / \sum_{y^{\prime}=1}^{g} p\left(\boldsymbol{x} \mid y^{\prime}\right)
$$

takes positive values, where $p(\cdot \mid y)$ is a class-conditional probability density of the category $C_{y}$.

AdaBoost aims to combine weak classifiers into a strong classifier. Let $\mathcal{F}=$ $\{f(\boldsymbol{x}, y)\}$ be a set of discriminant functions (weak classifiers). The loss of missclassification due to the function $f$ is assessed by the following exponential loss function:

$$
L\left(\boldsymbol{x}, y \mid y_{0}\right)=\exp \left[f(\boldsymbol{x}, y)-f\left(\boldsymbol{x}, y_{0}\right)\right]
$$

for $y \neq y_{0}, y=1, \ldots, g$, where $y_{0}$ be the true label of the feature vector $\boldsymbol{x}$. The loss function (4) is an extension of the exponential loss with binary class. The empirical risk is defined by

$$
R_{\mathrm{emp}}(f)=\frac{1}{n} \sum_{i=1}^{n} \sum_{y=1}^{g} \exp \left[f\left(\boldsymbol{x}_{i}, y\right)-f\left(\boldsymbol{x}_{i}, y_{i}\right)\right],
$$

where $\left(\boldsymbol{x}_{i}, y_{i}\right)$ are training data on the area $\mathcal{D}$.

Let $f$ and $F$ be discriminant functions (fixed). Then, the optimal coefficient $c$ 
which gives the minimum value of the empirical risk $R_{\mathrm{emp}}(F+c f)$ is denoted by $c_{*}$ :

$$
c_{*}=\underset{c \in \mathbb{R}}{\arg \min }\left\{R_{\mathrm{emp}}(F+c f)\right\} .
$$

If the function $f$ takes $0-1$ values like (2), the optimal coefficient $c_{*}$ can be expressed in the closed form.

If $f$ takes real values, there is no closed form to the optimal coefficient $c_{*}$. We will take an iterative procedure for the estimation. The convergence of the procedure is, however, very fast.

AdaBoost combines several discriminant functions so as to form a strong classifier. Now, we slightly extend Real AdaBoost for combining $T$ functions, where $T$ is a positive integer. Let $\mathcal{F}_{t}(\subset \mathcal{F})$ be a subset of discriminant functions for $t=1,2, \ldots, T$. (Possibly, $\mathcal{F}_{1}=\mathcal{F}_{2}=\cdots=\mathcal{F}_{T}$.) The procedure is as follows.

1. Find a weak discriminant function $f \in \mathcal{F}_{1}$ and the coefficient $c$ which minimize the empirical risk $R_{\mathrm{emp}}(c f)$ defined by the formula (5), say $f_{1}$ and $c_{1}$.

2. Consider the empirical risk $R_{\text {emp }}\left(c_{1} f_{1}+c f\right)$ with given $c_{1} f_{1}$ in the previous step. Then, find the optimal discriminant function $f \in \mathcal{F}_{2}$ and the coefficient $c$ which minimize the empirical risk, say $f_{2}$ and $c_{2}$.

3. This is repeated $T$-times and obtain the final discriminant function:

$$
F_{T}=c_{1} f_{1}+\cdots+c_{T} f_{T}
$$

4. A test vector $\boldsymbol{x}$ is classified into the label $\arg \max \left\{F_{T}(\boldsymbol{x}, y)\right\}$, where $F_{T}$ is defined in the above. $y \in\{1, \ldots, g\}$

We note that AdaBoost is based on a weighted majority vote principle.

\section{Neighborhoods and contextual classifiers}

As we have seen in Section II, AdaBoost requires multiple discriminant functions defined in the feature space. We will add contextual classifiers to the set of noncontextual classifiers so as to give contextual classification.

Let $d(i, j)$ be a distance between centers of two pixels $i$ and $j$ in the domain $\mathcal{D}$. Then, define a subset $U_{r}(i)$ of the area $\mathcal{D}$ with center $i$ and radius $r$ by

$$
U_{r}(i)=\{j \in \mathcal{D} \mid d(i, j)=r\} \text { for } r=0,1, \sqrt{2}, 2, \sqrt{5}, \ldots
$$

Note that the subset $U_{r}(i)$ is different from an ordinary neighborhood. See Fig. 1 for the subsets $U_{r}(i)$ for $r=0,1, \sqrt{2}, 2$. It is seen that $U_{0}(i)=\{i\}, U_{1}(i)$ is the first-order neighborhood of the pixel $i$, and $U_{1}(i) \cup U_{\sqrt{2}}(i)$ forms the secondorder neighborhood. Suppose that a statistical model is applied to the distribution in the feature space. Then, the posterior probability $p\left(y \mid \boldsymbol{x}_{i}\right)$ defined by (3) is a measure of confidence of the current classification. Such a measure is defined by machine learning techniques as well as statistical techniques. See [6-8] for posteriors 


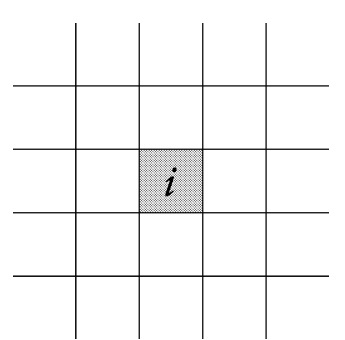

(a) $r=0$

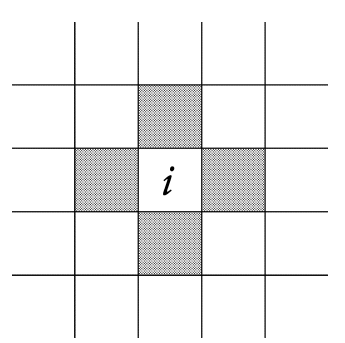

(b) $r=1$

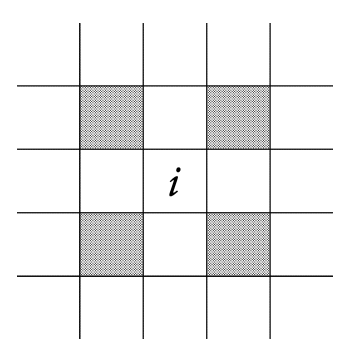

(c) $r=\sqrt{2}$

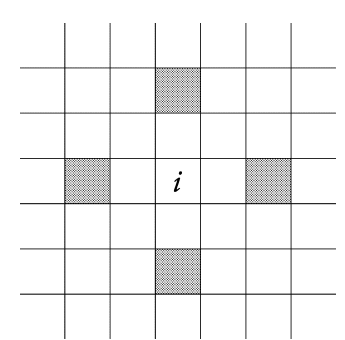

(d) $r=2$

Figure 1. Isotropic subsets $U_{r}(i)$ with a center pixel $i$ and radius $r$

introduced in SVM and AdaBoost.

Define the average of posterior probabilities in the subset $U_{r}(i)$ by

$$
\bar{p}_{r}\left(y \mid \boldsymbol{x}_{i}\right)=\left\{\begin{array}{cl}
\sum_{j \in U_{r}(i)} p\left(y \mid \boldsymbol{x}_{j}\right) /\left|U_{r}(i)\right|, & \text { if }\left|U_{r}(i)\right|>0 \\
0, & \text { otherwise }
\end{array}\right.
$$

for $r=0,1, \sqrt{2}, \ldots$, where $|S|$ denotes the cardinality of set $S$. Obviously, it holds that $\bar{p}_{0}\left(y \mid \boldsymbol{x}_{i}\right)=p\left(y \mid \boldsymbol{x}_{i}\right)$. Hence, noncontextual classification is done by the posterior $\bar{p}_{0}\left(y \mid \boldsymbol{x}_{i}\right)$, which is a strong classifier. If the spatial dependency of the categories exists, the averaged posteriors $\bar{p}_{1}\left(y \mid \boldsymbol{x}_{i}\right)$ in the adjacent pixels to the pixel $i$ also have information for classification. If the spatial dependency is strong, $\bar{p}_{r}\left(y \mid \boldsymbol{x}_{i}\right)$ with large $r$ is also useful. Thus, we adopt the average of the posteriors $\bar{p}_{r}\left(y \mid \boldsymbol{x}_{i}\right)$ as a classifier of the center pixel $i$.

Obviously, the importance of the posteriors for classifying the center pixel $i$ will be in the following order:

$$
\bar{p}_{0}\left(y \mid \boldsymbol{x}_{i}\right), \quad \bar{p}_{1}\left(y \mid \boldsymbol{x}_{i}\right), \quad \bar{p}_{\sqrt{2}}\left(y \mid \boldsymbol{x}_{i}\right), \cdots .
$$

The coefficients to the averaged posteriors $\bar{p}_{r}(\cdot \mid \cdot)$ can be tuned by minimizing the empirical risk (5).

Thus, candidates of contextual classifiers are as follows.

(1a) The averaged posteriors $\bar{p}_{r}\left(y \mid \boldsymbol{x}_{i}\right)$ as $(7)$ in the subset $U_{r}(i)$.

(1b) Threshholding of the function $\bar{p}_{r}\left(y \mid \boldsymbol{x}_{i}\right)$ by

$$
p_{r}\left(y \mid \boldsymbol{x}_{i}\right)= \begin{cases}1 & \text { if } y=\underset{0}{0} \text { otherwise. }\end{cases}
$$

(2a) Proportion of the labeled pixels in $U_{r}(i)$ :

$$
\bar{q}_{r}(y)=\left|\left\{j \in U_{r}(i) \mid y_{j}=y\right\}\right| /\left|U_{r}(i)\right| .
$$

(2b) Majority vote in $U_{r}(i)$ :

$$
q_{r}(y)= \begin{cases}1 & \text { if } y=\operatorname{argmax}_{y^{\prime}} \bar{q}_{r}\left(y^{\prime}\right) \\ 0 & \text { otherwise }\end{cases}
$$




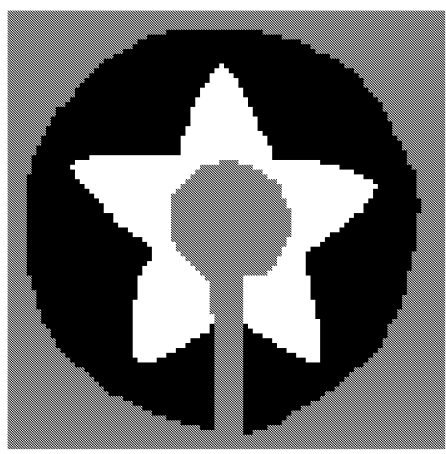

(a)

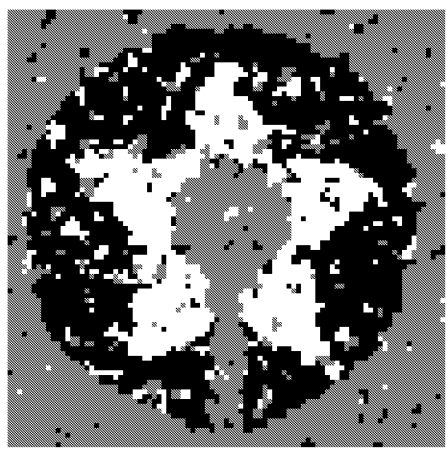

(d) $r=\sqrt{2}$

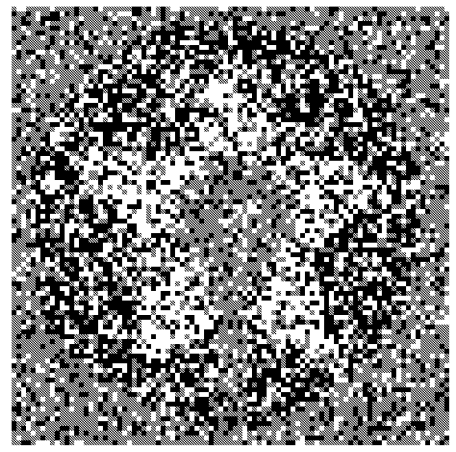

(b) $r=0$

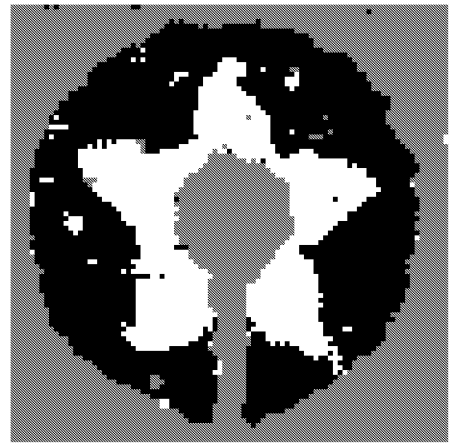

(e) $r=\sqrt{8}$

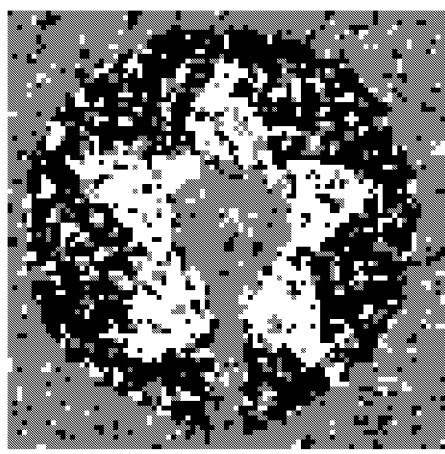

(c) $r=1$

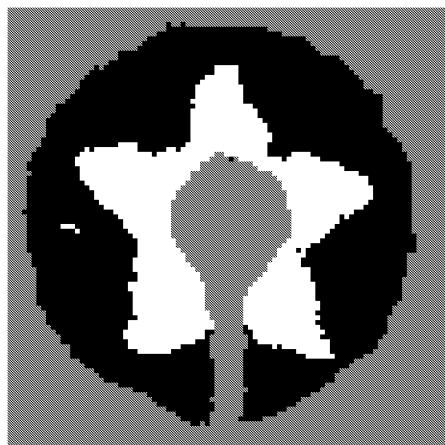

(f) $r=\sqrt{13}$

Figure 2. True labels (a) and estimated labels (b): by the linear discriminant function, and $(\mathrm{c}) \sim(\mathrm{f})$ : by Spatial AdaBoost based on $c_{0} \bar{p}_{0}+c_{1} \bar{p}_{1}+\cdots+c_{r} \bar{p}_{r}$

We here note that all the classifiers in the above are well-defined for the training data. However, the classifiers (2a) and (2b) cannot be defined by test data because test labels are unknown. If temporal estimates for test labels are available, the classifiers (2a) and (2b) are utilized. But, the classifiers supply only supplementary information.

Taking the importance of the posteriors, we propose to take $T$ subsets $\mathcal{F}_{1}, \ldots, \mathcal{F}_{T}$ of discriminant functions in Section 2 as follows.

$$
\mathcal{F}_{1}=\left\{\bar{p}_{0}\right\}, \mathcal{F}_{2}=\left\{\bar{p}_{1}\right\}, \mathcal{F}_{3}=\left\{\bar{p}_{\sqrt{2}}\right\}, \mathcal{F}_{4}=\left\{\bar{p}_{2}\right\}, \mathcal{F}_{5}=\left\{\bar{p}_{\sqrt{5}}\right\}, \cdots .
$$

Each subset consists of a single averaged posterior, and we get the final discriminant function of the form $c_{0} \bar{p}_{0}\left(y \mid \boldsymbol{x}_{i}\right)+c_{1} \bar{p}_{1}\left(y \mid \boldsymbol{x}_{i}\right)+\cdots+c_{r} \bar{p}_{r}\left(y \mid \boldsymbol{x}_{i}\right)$.

\section{Numerical experiments}

Our method is examined through multispectral images generated over the image (a) of Fig. 2 with three categories $(g=3)$. The labels 1,2 and 3 correspond to the colors black, white and grey. The numbers of pixels from the categories are respectively given by 3330, 1371 and 3580. We simulate four-dimensional spectral images $(m=$ 4) at each pixel of the true image (a) following multivariate normal distributions independently with mean vectors $\boldsymbol{\mu}(1)=\left(\begin{array}{llll}0 & 0 & 0 & 0\end{array}\right)^{T}, \boldsymbol{\mu}(2)=\left(\begin{array}{llll}1 & 1 & 0 & 0\end{array}\right)^{T} / \sqrt{2}, \boldsymbol{\mu}(3)=$ $(1.0498-0.637900)^{T}$ and with common variance-covariance matrix $\sigma^{2} E_{4}$, where $E_{4}$ denotes the identity matrix. Test data are similarly generated over the same image (a), and the normal distributions are used for driving the posteriors.

We set the subsets $\mathcal{F}_{t}$ of discriminant functions by the sequence (12). Hence, we 


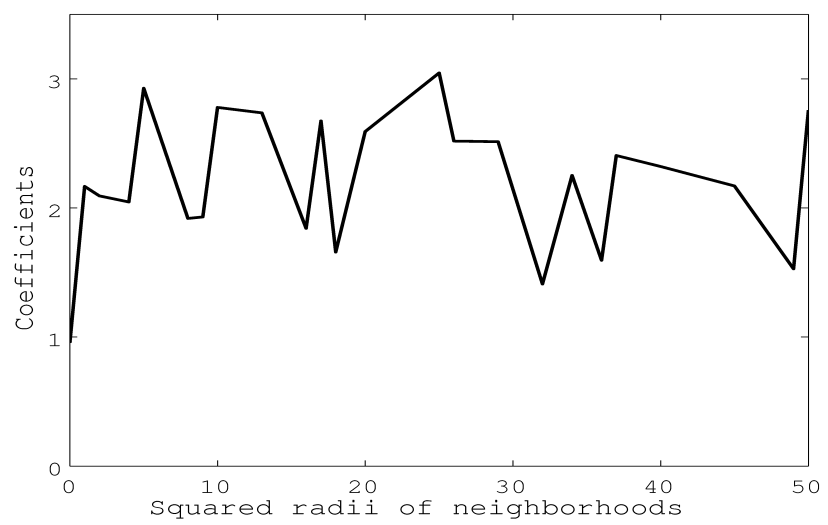

Figure 3. Estimated coefficients for the posteriors

applied the discriminant functions: $c_{0} \bar{p}_{0}, c_{0} \bar{p}_{0}+c_{1} \bar{p}_{1}, \cdots, c_{0} \bar{p}_{0}+c_{1} \bar{p}_{1}+\cdots+c_{\sqrt{50}} \bar{p}_{\sqrt{50}}$ to test data, where the coefficient $c_{r}$ is sequentially tuned by minimizing the empirical risk.

Fig. 3 plots the coefficients $c_{0}, c_{1}, \ldots, c_{\sqrt{50}}$ against radius $r$ in the case $\sigma^{2}=1$. Magnitude of the coefficients give a reliability of the corresponding classifiers. The coefficient $c_{0}$ is not large, and this is ascertained by the fact that the error rate due to $\bar{p}_{0}$ (the noncontextual classifier) is $41.75 \%$.

Fig. 4 show training and test error rates due to the classifiers $c_{0} \bar{p}_{0}+c_{1} \bar{p}_{1}+\cdots+c_{r} \bar{p}_{r}$ for $r=0,1, \ldots, \sqrt{50}$ in the case with error variance $\sigma^{2}=1$. The trainig error (a) shows that the neighborhood information reduces the error rate rapidly, and it is stable even if the radius $r$ is too large. The test error attains the minimum value at $r^{2}=13$

Fig. 2 (b) is the classified image obtained by the posterior $\bar{p}_{0}$, which is equivalent to the linear discriminant function. The classified images are getting closer to the true label (a) as the radius $r$ is getting larger.

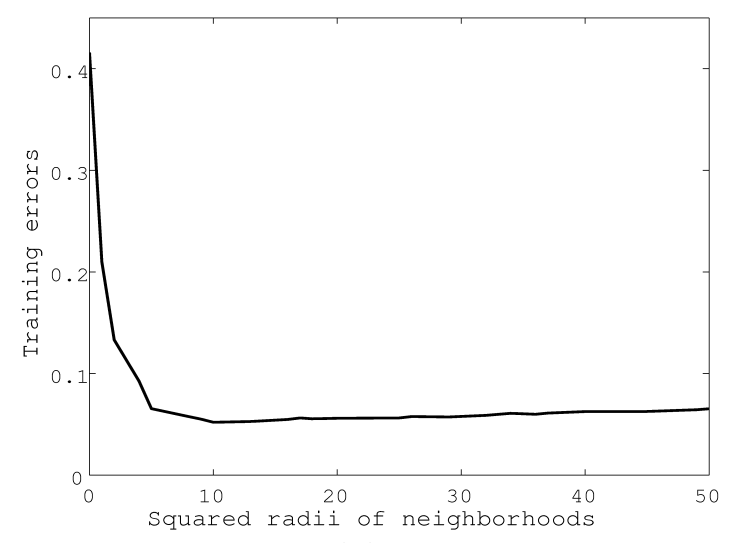

(a)

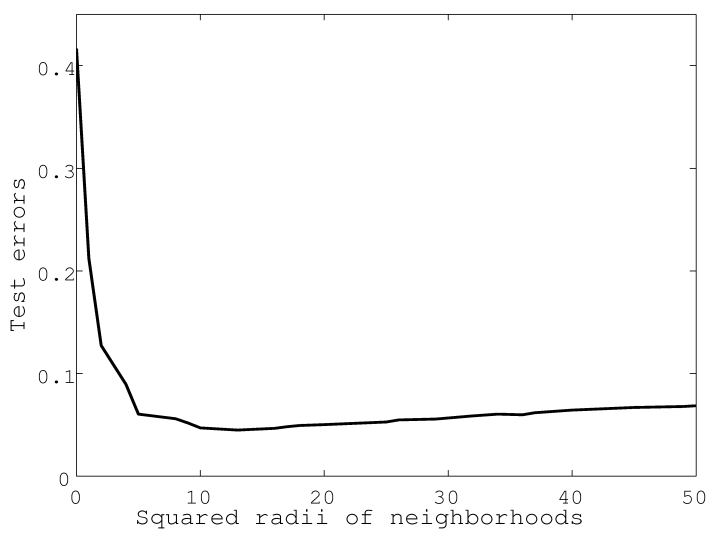

(b)

Figure 4. Error rates against squared radius $r^{2}$ for training data (a) and test data (b) due to the discriminant functions $c_{0} \bar{p}_{0}+c_{1} \bar{p}_{1}+\cdots+c_{r} \bar{p}_{r}$ 
TABLE 1. Error rates (\%) of classification results based on Gaussian MRF and Spatial Real AdaBoost

\begin{tabular}{|c||c|c||c|c||c|c|}
\hline \multirow{2}{*}{ radius } & \multicolumn{5}{c|}{ Distributions of the categories : $\mathrm{N}_{4}\left(\boldsymbol{\mu}(g), \sigma^{2} E\right)$} \\
\cline { 2 - 7 }$r$ & \multicolumn{3}{c||}{$\sigma^{2}=1$} & \multicolumn{2}{|c|}{$\sigma^{2}=4$} \\
\cline { 2 - 7 } & GMRF & CPU & AdaBoost & CPU & GMRF & AdaBoost \\
\hline \hline 0 & 41.75 & - & 41.75 & - & 54.56 & 54.56 \\
\hline 1 & 10.68 & 210 & 21.46 & 1.66 & 40.55 & 41.03 \\
$\sqrt{2}$ & 5.01 & 409 & 12.91 & 1.70 & 17.14 & 34.15 \\
2 & 3.57 & 617 & 8.98 & 1.64 & 12.04 & 29.11 \\
$\sqrt{5}$ & 3.23 & 852 & 6.09 & 2.00 & 10.04 & 22.50 \\
$\sqrt{8}$ & 4.61 & 1117 & 5.62 & 1.83 & 10.18 & 20.88 \\
3 & 5.12 & 1402 & 5.17 & 1.81 & 10.19 & 19.26 \\
$\sqrt{10}$ & 6.10 & 1716 & 4.64 & 2.24 & 11.05 & 16.50 \\
$\sqrt{13}$ & 9.52 & 2082 & 4.46 & 2.20 & 19.41 & 14.88 \\
4 & 11.70 & 2481 & 4.64 & 1.97 & 18.51 & 14.31 \\
$\sqrt{17}$ & 13.56 & 2904 & 4.79 & 2.27 & 20.66 & 13.48 \\
$\sqrt{18}$ & 21.78 & 3371 & 4.87 & 2.13 & 21.46 & 13.28 \\
$\sqrt{20}$ & 30.58 & 3376 & 5.00 & 2.41 & 22.79 & 12.33 \\
\hline
\end{tabular}

Table 1 compares error rates due to Gaussian MRF-based (GMRF) and the proposed discriminant functions in two cases: $\sigma^{2}=1,4$. Each row is corresponding to GMRF with the neighborhood system $U_{1}(i) \cup U_{\sqrt{2}}(i) \cup \cdots \cup U_{r}(i)$ and the discriminant function $c_{0} \bar{p}_{0}+c_{1} \bar{p}_{1}+\cdots+c_{r} \bar{p}_{r}$. The third and fifth columns show CPU time (seconds) required to GMRF and the proposed method respectively. GMRF needs much time for tuning the dependency parameter [5], whereas the the proposed method determines the coefficient sequentially with several iterations. It is shown that the proposed method is very fast procedure compared with the ordinary MRF-based classifier, and that both the classifiers show similar performance.

\section{Conclusion}

Real AdaBoost is introduced to provide contextual image classification. Weak classifiers based on posteriors on subsets of neighbors are proposed. The proposed method is flexible in the following two points. One is that various types of posteriors obtained by machine learning techniques as well as statistical models can be implemented. The other is in the definitions of the neighborhoods. In Section II, isotropic neighborhoods of a center pixel are considered (Fig. 1). In nonisotropic image case, directed neighborhoods would be useful, see Fig. 5. This means that the proposed method would be also applicable to image classification with texture.

Features of the proposed method are as follows.

- Various types of posteriors can be implemented.

- Various types of neighborhoods can be implemented.

- The method requires much less computation time and shows similar performance to MRF-based classifiers.

Selection of types of posteriors and neighborhoods is a new problem. Selection of the number of classifiers is also an old and new problem. 

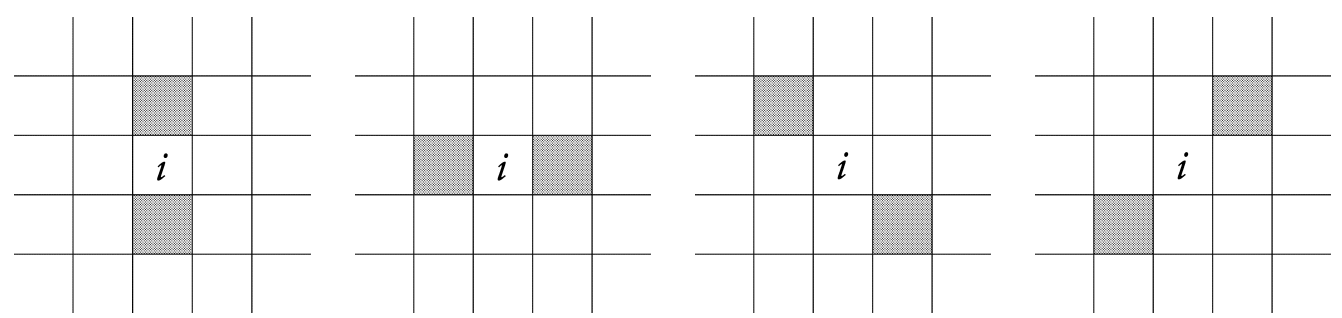

Figure 5. Directed subsets with a center pixel $i$ and radius $r=1, \sqrt{2}$

References

[1] Freund, Y. and Schapire, R.E. (1997): A decision-theoretic generalization of online learning and an application to boosting. Journal of Computer and System Sciences, vol. 55(1), 119-139.

[2] Vapnik, V. N. (2000): The Nature of Statistical Learning Theory, Springer, New York.

[3] Hastie, T., Tibshirani, R. and Friedman, J. (2001): The Elements of Statistical Learning : Data Mining, Inference, and Prediction, Springer, New York.

[4] Takenouchi, T. and Eguchi, S. (2004): Robustifying AdaBoost by adding the naive error rate, Neural Computation, vol. 16, 767-787.

[5] Nishii, R. (2003): A Markov random field-based approach to decision level fusion for remote sensing image classification. IEEE Transactions on Geoscience and Remote Sensing, vol. 41(10), 2316-2319.

[6] Kwok, J.T. (2000): The evidence framework applied to support vector machine IEEE Transactions on Neural Networks, vol. 11(5), 1162-1173.

[7] Friedman, J., Hastie, T. and Tibshirani, R. (2000): Additive logistic regression: a statistical view of boosting (with discussion), Annals of Statistics, vol. 28, $337-407$.

[8] Roth, V. (2001): Probabilistic discriminative kernel classifiers for multiclass problems. Deutsche Arbeitsgemeinschaft für Mustererkennung(DAGM) symposium, 246-253. 


\section{List of MHF Preprint Series, Kyushu University 21st Century COE Program Development of Dynamic Mathematics with High Functionality}

\section{MHF2003-1 Mitsuhiro T. NAKAO, Kouji HASHIMOTO \& Yoshitaka WATANABE}

A numerical method to verify the invertibility of linear elliptic operators with applications to nonlinear problems

MHF2003-2 Masahisa TABATA \& Daisuke TAGAMI

Error estimates of finite element methods for nonstationary thermal convection problems with temperature-dependent coefficients

MHF2003-3 Tomohiro ANDO, Sadanori KONISHI \& Seiya IMOTO

Adaptive learning machines for nonlinear classification and Bayesian information criteria

MHF2003-4 Kazuhiro YOKOYAMA

On systems of algebraic equations with parametric exponents

MHF2003-5 Masao ISHIKAWA \& Masato WAKAYAMA

Applications of Minor Summation Formulas III, Plücker relations, Lattice paths and Pfaffian identities

MHF2003-6 Atsushi SUZUKI \& Masahisa TABATA

Finite element matrices in congruent subdomains and their effective use for large-scale computations

MHF2003-7 Setsuo TANIGUCHI

Stochastic oscillatory integrals - asymptotic and exact expressions for quadratic phase functions -

MHF2003-8 Shoki MIYAMOTO \& Atsushi YOSHIKAWA

Computable sequences in the Sobolev spaces

MHF2003-9 Toru FUJII \& Takashi YANAGAWA

Wavelet based estimate for non-linear and non-stationary auto-regressive model

MHF2003-10 Atsushi YOSHIKAWA

Maple and wave-front tracking - an experiment

MHF2003-11 Masanobu KANEKO

On the local factor of the zeta function of quadratic orders

MHF2003-12 Hidefumi KAWASAKI

Conjugate-set game for a nonlinear programming problem 
MHF2004-1 Koji YONEMOTO \& Takashi YANAGAWA

Estimating the Lyapunov exponent from chaotic time series with dynamic noise

MHF2004-2 Rui YAMAGUCHI, Eiko TSUCHIYA \& Tomoyuki HIGUCHI

State space modeling approach to decompose daily sales of a restaurant into time-dependent multi-factors

MHF2004-3 Kenji KAJIWARA, Tetsu MASUDA, Masatoshi NOUMI, Yasuhiro OHTA \& Yasuhiko YAMADA

Cubic pencils and Painlevé Hamiltonians

MHF2004-4 Atsushi KAWAGUCHI, Koji YONEMOTO \& Takashi YANAGAWA

Estimating the correlation dimension from a chaotic system with dynamic noise

MHF2004-5 Atsushi KAWAGUCHI, Kentarou KITAMURA, Koji YONEMOTO, Takashi YANAGAWA \& Kiyofumi YUMOTO

Detection of auroral breakups using the correlation dimension

MHF2004-6 Ryo IKOTA, Masayasu MIMURA \& Tatsuyuki NAKAKI

A methodology for numerical simulations to a singular limit

MHF2004-7 Ryo IKOTA \& Eiji YANAGIDA

Stability of stationary interfaces of binary-tree type

MHF2004-8 Yuko ARAKI, Sadanori KONISHI \& Seiya IMOTO

Functional discriminant analysis for gene expression data via radial basis expansion

MHF2004-9 Kenji KAJIWARA, Tetsu MASUDA, Masatoshi NOUMI, Yasuhiro OHTA \& Yasuhiko YAMADA

Hypergeometric solutions to the $q$ - Painlevé equations

MHF2004-10 Raimundas VIDŪNAS

Expressions for values of the gamma function

MHF2004-11 Raimundas VIDŪNAS

Transformations of Gauss hypergeometric functions

MHF2004-12 Koji NAKAGAWA \& Masakazu SUZUKI

Mathematical knowledge browser

MHF2004-13 Ken-ichi MARUNO, Wen-Xiu MA \& Masayuki OIKAWA

Generalized Casorati determinant and Positon-Negaton-Type solutions of the Toda lattice equation

MHF2004-14 Nalini JOSHI, Kenji KAJIWARA \& Marta MAZZOCCO

Generating function associated with the determinant formula for the solutions of the Painlevé II equation 
MHF2004-15 Kouji HASHIMOTO, Ryohei ABE, Mitsuhiro T. NAKAO \& Yoshitaka WATANABE

Numerical verification methods of solutions for nonlinear singularly perturbed problem

MHF2004-16 Ken-ichi MARUNO \& Gino BIONDINI

Resonance and web structure in discrete soliton systems: the two-dimensional Toda lattice and its fully discrete and ultra-discrete versions

MHF2004-17 Ryuei NISHII \& Shinto EGUCHI

Supervised image classification in Markov random field models with Jeffreys divergence

MHF2004-18 Kouji HASHIMOTO, Kenta KOBAYASHI \& Mitsuhiro T. NAKAO

Numerical verification methods of solutions for the free boundary problem

MHF2004-19 Hiroki MASUDA

Ergodicity and exponential $\beta$-mixing bounds for a strong solution of Lévydriven stochastic differential equations

MHF2004-20 Setsuo TANIGUCHI

The Brownian sheet and the reflectionless potentials

MHF2004-21 Ryuei NISHII \& Shinto EGUCHI

Supervised image classification based on AdaBoost with contextual weak classifiers 